

\title{
APLICAÇÃO DO CUSTEIO POR ATIVIDADES AO PROCESSO DE PREPARAÇÃO DE PROPOSTAS POR EMPRESA DE CONSTRUÇÃO
}

\author{
SANCHES, Rodrigo (1); ISATTO, Eduardo (2); GUANABARA, \\ Guanabara (3)
}

(1) UFRGS, e-mail: rodrigo_sanches4@hotmail.com, (2)UFRGS, e-mail: isatto@ufrgs.br,

(3) UFRGS, e-mail: matheuskguanabara@hotmail.com

\begin{abstract}
Resumo
As construções públicas representam importante papel no mercado de construção brasileiro. No entanto, é necessária a inserção das empresas atuantes neste mercado, em um processo competitivo de concorrência por obras (licitação), que é estratégica e economicamente importante para tais empresas. Portanto, a precisão na determinação dos custos deste processo de gestão é extremamente importante para estas empresas controlarem os processos de licitações, gerando informações importantes para a tomada de decisão. No entanto, por suas características produtivas e vários custos indiretos envolvidos no processo, há uma grande dificuldade no controle e alocação destes custos. Este artigo trata da aplicação do custeio baseado em atividades (custeio $\mathrm{ABC}$ ) ao processo de orçamentos de uma empresa que opera no setor de execução de obras públicas. No estudo, é descrito o processo de implementação, e realizada a análise crítica dos resultados obtidos, comparando a metodologia de custeio $\mathrm{ABC}$ com o sistema de custeio tradicional.
\end{abstract}

Palavras-chave: custeio por atividades, licitações, obras públicas

\begin{abstract}
The construction of public buildings plays an important role in the Brazilian construction market. However, it is necessary the inclusion of companies that active on this market in a competitive bidding process for the construction of these buildings, which is strategically and economically important for those companies. Therefore, the accuracy in determining the costs of this management process is extremely important for any organization to control the bidding process, generating important information for decision-making. However, for their productive characteristics and various indirect costs involved in the construction process, there is great difficulty in controlling and allocating these costs. This paper deals with the application of activity-based costing (ABC costing) in the budgets process of a company that operates in the execution of public buildings sector. This study describes the implementation process, and makes the critical analyze of the results, comparing the ABC costing methodology and traditional costing system.
\end{abstract}

Keywords: activity costing, procurement

\section{INTRODUÇÃO}

As obras públicas contribuem para atender parcela significativa da demanda habitacional do país e de obras de infraestrutura, além de desempenhar papel fundamental para o desempenho financeiro nacional pela geração de empregos e aquecimento da economia. A contratação das obras públicas se dá através de licitações, regida pela Lei 8.666/93, em um processo segundo o qual é escolhida a empresa que oferece a proposta considerada mais vantajosa ao Poder Público, geralmente entendida a 
de menor preço (BRASIL, 1993). Como decorrência, a apuração precisa dos custos por parte das empresas proponentes passa a ser crítica, uma vez que a competição ocorre fundamentalmente com base nos custos orçados. Entretanto, ainda hoje muitas empresas fazem uso da sistemática tradicional de custeio, baseadas na contabilidade financeira, a qual é duramente criticada por Johnson e Kaplan (1993), os quais sustentam a perda da sua relevância para a tomada de decisões na empresa. Nakagawa (2001) afirma que o fato dos sistemas de custos considerados tradicionais não atenderem as necessidades das organizações já é bastante reconhecido, estimulando a criação de novos sistemas e/ou métodos para custeio. Kaplan e Cooper (1998) defendem a necessidade de métodos e sistemas inovadores de custeio para ocupar o papel anteriormente desempenhado pela contabilidade financeira no apoio a tomada de decisão gerencial, dentre eles o Custeio Baseado em Atividades (Activity-Based Costing, ou ABC).

$\mathrm{O}$ método $\mathrm{ABC}$ tem como fundamento básico a busca do princípio da causa, ou seja, procura identificar de forma clara, por meio de rastreamento, o agente causador do custo, para the imputar o valor (MARTINS, 2003).

Há dois propósitos para aplicação do ABC (Brimson, 1994). A primeira finalidade é a de alocar os custos aos produtos, evitando as distorções que ocorrem pelo fato do custeio tradicional combinar todos os custos indiretos em um único centro de custo, o que implica em um critério único de rateio (geralmente ligado ao trabalho) para todos esses custos, independentemente da sua diversidade. $\mathrm{O}$ método $\mathrm{ABC}$ tenta reduzir tais distorções na medida em que aloca primeiramente os custos às atividades (através de direcionadores de custos para as atividades) e somente depois direcionando os mesmos, através dos direcionadores de custos aos produtos. A segunda finalidade é visando à melhoria dos processos, de forma a atuar nas causas dos custos, reduzindo-os. Kim e Ballard (2001) enfatizam esta segunda finalidade no que se refere a aplicação do ABC na construção, como forma de minimizar o desperdício ou atividades que não agregam valor, proporcionando uma visão de processo.

O presente trabalho tem como objetivo dissertar sobre a aplicação do custeio ABC para o processo de alocação de custos do processo de elaboração de propostas de preços para licitações. Tal processo tem uma participação relevante nos custos gerais da empresa. No que se refere à alocação dos custos do processo às obras, a aplicação do $\mathrm{ABC}$ pode evitar distorções no processo de rateio dos custos indiretos, contribuir para melhorar as estimativas de custos realizadas pela empresa construtora durante a fase de licitação de obras, reduzindo as distorções na alocação dos custos relativos a este processo. Já no que diz respeito a uma melhor compreensão do processo e dos fatores que afetam os custos a ele associados, o ABC pode fornecer subsídios para atuar de forma eficaz sobre tais custos, influenciando-os de forma a tornar os preços ofertados mais competitivos.

\section{ANÁLISE GERENCIAL DE CUSTOS}

Um sistema de contabilidade utiliza todos os eventos econômicos e transações, como vendas, compra de materiais, etc., e os transforma em informações úteis para gerentes, representantes de vendas, diretores e demais interessados. Essa transformação se baseia no correto registro desses eventos, dividindo-os em categorias pertinentes às necessidades dos usuários do sistema (materiais, mão de obra, equipamentos, entre outros), resumindo os dados coletados. Assim, o acesso às informações é simplificado e possibilita melhor compreensão para todos os usuários. Posteriormente, possibilita a análise do material gerado, fazendo comparações entre os resultados de diversos períodos e previsões para os períodos futuros. Uma correta compreensão desses dados é 
de suma importância para auxiliar na tomada de decisões estratégicas. (HORNGREN et al., 2012).

Existem dois focos básicos para o registro dos eventos econômicos: a contabilidade financeira e a contabilidade gerencial. A primeira visa registrar eventos financeiros e fiscais, alocando os custos de produção entre produtos vendidos e em estoque, fornecendo relatórios auditáveis para o público externo da empresa, investidores, entidades do governo, bancos, entre outros, sempre respeitando os Princípios Contábeis Geralmente Aceitos (PCGA) e as demais legislações pertinentes (KHOURY e ANCELEVICZ, 2000)

Já a contabilidade gerencial, tem por objetivo medir, analisar e difundir as informações, financeiras ou não, que auxiliem na tomada de decisão dos gerentes, a fim de atingir as metas da organização. Ressaltando-se que por ter como público-alvo o público interno da empresa não é necessário que siga os PCGA (HORNGREN et al., 2012). Tais autores afirmam que a contabilidade gerencial deve levar em conta dois fatores importantes: (a) como a informação pode auxiliar os gerentes a tomarem melhores decisões; (b) se os benefícios gerados por ela compensam os custos de sua geração.

Através da análise gerencial dos custos, podemos ter uma melhor visão do processo estudado, analisando os recursos e atividades envolvidas, identificando pontos de maior ou menor eficiência e traçando estratégias para a diminuição dos custos. Ainda é possível perceber as atividades que consomem mais recursos e ter uma visão mais global e completa do processo, proporcionando oportunidades de melhoria na gestão.

\subsection{Custos diretos e indiretos}

A classificação dos custos em diretos e indiretos tem como principal preocupação a correta alocação dos recursos empregados aos objetos de custo, dentre eles os produtos finais. Os custos diretos de um objeto de custo são aqueles relacionados ao objeto de custo em particular, e podem ser a ele atribuídos de uma maneira economicamente viável. Já os custos indiretos de um objeto não podem ser a ele atribuídos de uma maneira economicamente viável e, portanto são a ele relacionados através de algum critério de rateio (HORNGREN et al., 2012). Quando os objetos de custo são iguais ou muito semelhantes, a alocação dos custos indiretos aos mesmos é simples: basta dividilos pelas quantidades produzidas de cada um dos produtos. No entanto, isto não ocorre quando os objetos são distintos, podendo conduzir a erros de alocação e, como consequência, distorções no cálculo do custo total destes objetos de custo. Este problema é particularmente relevante na medida em que aumenta a participação dos custos indiretos em relação ao custo total.

\subsection{Sistema de custeio abc}

O custeio ABC surgiu em meados dos anos 80 com o objetivo de suprir a necessidade de informações precisas sobre o custo de recursos de produtos. Esse sistema passa a ser de grande valia para as tomadas de decisões gerenciais e para o processo de melhoria da empresa. Ele ainda é apontado por Horngren et al. (2012) como uma das melhores ferramentas para refinar um sistema de custo, por identificar atividades individuais como os objetos de custo fundamentais, auxiliando na tomada de decisões estratégicas.

Conforme Mauad e Pamplona (2002), à medida que as empresas adotam uma filosofia pela qual a gerência do processo do negócio é a chave para a gerência de toda a empresa, a ligação entre atividades e custos assume importância crucial para tornar a contabilidade gerencial de grande valor para os gerentes de hoje. Dessa forma, o sistema 
de custeio $\mathrm{ABC}$ permite melhor visualização dos custos através da análise das atividades executadas dentro da empresa e suas respectivas relações com os objetos de custos. Com seu poder de assinalar as "causas" que levam ao surgimento dos custos, o $\mathrm{ABC}$ permite aos gerentes uma atuação mais seletiva e eficaz sobre o comportamento dos custos da organização.

$\mathrm{O}$ custeio $\mathrm{ABC}$ visa reduzir as distorções geradas pelo rateio arbitrário dos custos indiretos através da obtenção de informações mais precisas dos custos dos produtos produzidos e serviços prestados e da identificação dos custos relativos das atividades e as razões dessas atividades serem empreendidas. Ele parte do princípio de que as atividades geram custos e de que os produtos produzidos e os serviços prestados causam demanda por essas atividades. $\mathrm{O}$ ABC utiliza as atividades desenvolvidas nos processos de operação da empresa como ligação dos custos indiretos aos objetos de custos, definidos como produtos, linhas de produtos, serviços, clientes, etc (KHOURY e ANCELEVICZ, 2000).

Figura 1 - Fluxo de custos no Sistema de Custeio ABC



Calesso (2010) ainda aponta diversas qualidades do método de custeio ABC: (a) informação gerenciais mais fidedignas; (b) menor necessidade de rateios arbitrários; (c) obriga a implantação e manutenção de sistemas internos de controle; (d) proporciona melhor visualização dos fluxos dos processos; (e) identificar quais as atividades consomem mais recursos; (f) possibilita a identificação e eliminação de atividades que não agregam valor ao produto.

Para aplicar o sistema de custo baseado em atividades, Horngren et al. (2012) sugere que sejam seguidas as três diretrizes principais para refinar o sistema de custeio: identificar a maior quantidade possível de custos diretos, aumentar a quantidade de grupos de custos indiretos até que cada um seja mais homogêneo, ou seja, que os grupos representem elementos cujos custos foram gerados pelos mesmos motivos, e usar os motivos que geraram os custos indiretos para fazer sua vinculação com as atividades.

Horngren et al. (2012) aponta ainda, sete etapas que devem ser adotadas para a implementação do sistema: (a) identificar os produtos que são os objetos de custo escolhidos; (b) identificar os custos diretos do produto; (c) selecionar a forma de distribuir os custos indiretos com base em seus processos geradores; (d) identificar cada um dos custos indiretos associados ao processo; (e) calcular o custo por unidade de cada um dos grupos de custos indiretos; (f) calcular o custo indireto de cada produto; (g) calcular o custo total do produto somando os custos diretos e indiretos envolvidos.

\section{PROCESSO DE ELABORAÇÃO DE PROPOSTAS PARA LICITAÇÕES}

As licitações públicas são procedimentos baseados em princípios constitucionais (legalidade, impessoalidade, moralidade, etc.), onde o objetivo é proporcionar a 
administração, a aquisição, a venda ou uma prestação de serviço de forma vantajosa, ou seja, menor custo, menor prazo e maior qualidade. Isto é a chamada "eficiência contratória" (ALTOUNIAN, 2012).

Altounian (2012) afirma ainda, que no processo licitatório, as empresas concorrentes elaboram suas propostas, que devem atender às especificações legais publicadas nos editais. Neste caso, há a comparação entre propostas e a empresa que oferecer maiores vantagens ao governo será acolhida para o fornecimento do produto, serviço ou bem de consumo.

O critério final que as instituições que fazem licitações utilizam para definir o vencedor da concorrência na licitação é o menor preço, uma vez que os demais critérios são baseados em fatos históricos referentes à experiência da empresa quanto a sua atuação no mercado e precisam ser comprovados antes da análise de custos. (ALTONIAN, 2012)

Para tanto, a empresa necessita elaborar bem suas propostas, com orçamentos de execução inferiores ao edital e aos orçamentos das concorrentes, uma vez que a demanda de serviço dela está diretamente ligado aos ganhos em processos licitatórios. E, caso ela perca as licitações concorrentes, os ônus de elaboração de propostas são de responsabilidade exclusiva da empresa.

\section{ESTUDO DE CASO}

\subsection{Caracterização da empresa e do processo}

O estudo de caso de que trata este artigo foi realizado em uma empresa onde foi desenvolvido atua no Sul do País, com sede em Porto Alegre e desempenha suas atividades no estado do Rio Grande do Sul e Santa Catarina. Esta é classificada como de pequeno-médio porte com faturamento anual de aproximadamente R $\$ 10.000 .000,00$. Ela está inserida no mercado de execução de obras públicas e a única forma utilizada para contratar novas obras é a participação em concorrências públicas, as licitações.

$\mathrm{O}$ processo de concorrência em licitações como observado na empresa encontra-se representado na Figura 2. Como forma de assegurar um bom conhecimento de mercado e uma precisa avaliação de custos, os quais são fatores essenciais para a sobrevivência da empresa, esta possui um setor especializado e dedicado exclusivamente à prospecção de novas obras e acompanhamento do mercado, identificando quais as obras de interesse, bem como seus principais concorrentes.

Figura 2 - Representação do processo de concorrência em licitações

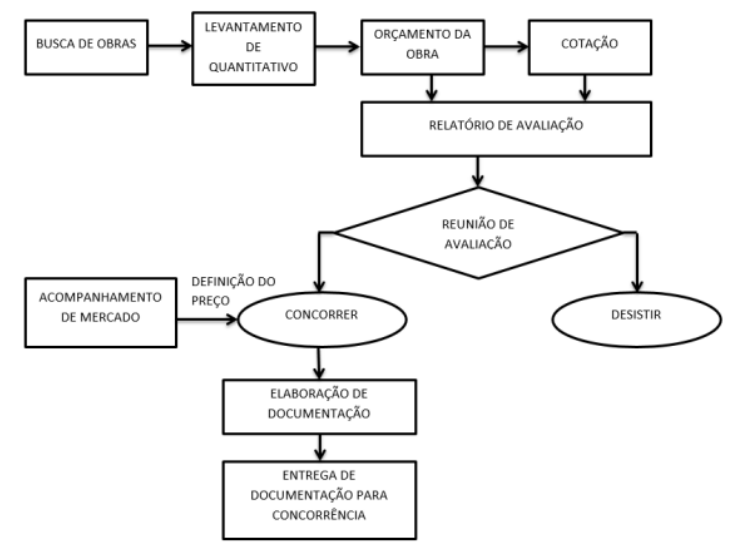


As fontes de evidência utilizadas nesta pesquisa foram entrevistas e a análise de documentos contábeis da empresa construtora. Nas informações financeiras, foi empregado um fator multiplicador em relação aos números coletados, com o intuito de manter sigilo dos mesmos, aplicou-se um fator multiplicador.

\subsection{Aplicação do custeio $\mathrm{ABC}$ ao processo adotado pela empresa}

O processo de elaboração de propostas acarretava custos relevantes para a empresa, que mereciam ser bem apurados com o intuito de fornecer informações precisas $\mathrm{e}$ necessárias para a boa gestão. No entanto, algumas dificuldades quanto à alocação desses custos nas obras puderam ser constatadas.

A primeira dizia respeito à dificuldade da correta alocação dos custos indiretos. Algumas atividades que compunham o processo de preparação de propostas eram bastante complexas, necessitando a participação de gerentes e diretores da empresa para tomada de decisões estratégicas. Como tais custos eram difíceis de serem alocados de maneira direta às obras, a empresa optou por adotar um critério bastante simples para distribuí-los pelas mesmas: o rateio dos custos anuais gerados pelo setor era realizado segundo o total de área $\mathrm{em} \mathrm{m}^{2}$ das obras avaliadas no período. Já existia na ocasião a percepção por parte da empresa que as obras orçadas diferiam muito entre si em termos do esforço empregado e que o critério de rateio por $\mathrm{m}^{2}$ ocasionaria distorções no processo de atribuição dos custos às obras.

Outro fato complicador quanto a esta alocação era que nem todas as obras avaliadas resultavam em contratações ao final. Havia casos de propostas que eram elaboradas e, ao final, não obtinham o êxito da contratação, assim como propostas que eram abandonadas ainda durante o processo, por questões diversas, tais como a baixa rentabilidade esperada caso houvesse a contratação.

Os dados da empresa indicavam que no ano de 2012 o setor de orçamentos foi responsável por um custo de $\mathrm{R} \$ 247.693,97$, tendo sido analisadas 50 licitações, totalizando uma área de $61.860 \mathrm{~m}^{2}$. Desta forma, segundo os controles da empresa, foram gastos aproximadamente $\mathrm{R} \$ 4,00$ por $\mathrm{m}^{2}$ analisado. Na Tabela 1 são apresentados estes dados, distribuídos segundo o tipo de obra (licitações avaliadas e desistidas, licitações concorridas e perdidas e concorrência licitações concorridas e vencidas), e o valor do rateio atribuído a cada um deles.

Tabela 1 - Custo por perfil de participação em um ano

\begin{tabular}{|l|c|r|}
\hline \multicolumn{1}{|c|}{ Perfil de participação } & \multicolumn{1}{c|}{$\mathbf{M}^{\mathbf{2}}$} & CUSTO \\
\hline Licitação Vencida & 19690 & $\mathrm{R} \$ 80.877,95$ \\
\hline Lic. Concorridas e perdidas & 23347 & $\mathrm{R} \$ 95.899,32$ \\
\hline Lic. Avaliadas e desistidas & 18823 & $\mathrm{R} \$ 77.316,70$ \\
\hline TOTAL & 61860 & $\mathrm{R} \$ 254.093,97$ \\
\hline
\end{tabular}

\subsubsection{Identificação dos custos dos recursos:}

Partindo deste ponto, para análise de custeio $\mathrm{ABC}$ é necessário identificar os recursos utilizados no processo e os custos de cada recurso nos períodos, como demonstrado na Tabela 2. 
Tabela 2 - Recursos utilizados e custos associados em um ano

\begin{tabular}{|c|c|l|r|}
\hline Cód. Recurso & Quant & \multicolumn{1}{|c|}{ Recurso } & Custo no período \\
\hline 1 & 1 & Gerente & $\mathrm{R} \$ 78.542,00$ \\
\hline 2 & 1 & Engenheiro & $\mathrm{R} \$ 48.195,00$ \\
\hline 3 & 1 & Comprador & $\mathrm{R} \$ 25.700,00$ \\
\hline 4 & 1 & Func. Adm. & $\mathrm{R} \$ 13.584,00$ \\
\hline 5 & 1 & Estagiário & $\mathrm{R} \$ 5.700,00$ \\
\hline 6 & 2 & Software & $\mathrm{R} \$ 6.400,00$ \\
\hline 7 & 3 & Veículo & $\mathrm{R} \$ 62.317,97$ \\
\hline 8 & 4 & Plotagem & $\mathrm{R} \$ 8.315,00$ \\
\hline 9 & 1 & Telefone & $\mathrm{R} \$ 5.340,00$ \\
\hline TOTAL & & & $\mathrm{R} \$ 254.093,97$ \\
\hline
\end{tabular}

\subsubsection{Alocação dos custos às atividades}

Em seguida é necessário identificar quais as atividades envolvidas no processo e a forma como estes recursos são utilizados para execução de cada atividade. É necessário, então, a atribuição de direcionadores de custo de cada recurso para as atividades, como mostrado na Tabela 3. Em todos os recursos da Tabela 2 o direcionador de custo adotado foi a percepção dos envolvidos quanto a parcela de tempo dedicada a cada atividade, à exceção do recurso 7 (veículo), onde foi utilizada a distância.

A partir da definição dos direcionadores de custos dos recursos para as atividades, devese medir os quantitativos de cada recurso utilizado para a realização de cada tarefa por ele executada, como mostrado na Tabela 4. Para obtenção dos custos de cada uma das atividades no período, deve-se direcionar o custo das atividades para cada perfil de licitação. Para tanto, é necessário atribuir os direcionadores para cada atividade, sendo necessários os dados de $\mathrm{n}^{\circ}$ de obras avaliadas no período, distância da obra para a sede da empresa em quilômetros, área da obra $\mathrm{em}^{2}$ e preço orçado em milhões.

Foram então utilizados os direcionadores de custo para as atividades, onde dividiu-se os custos de cada atividade pela quantidade dos direcionadores totais, obtendo o custo unitário por atividade, conforme mostra a Tabela 4.

Tabela 3 - Direcionamento de custos dos recursos às atividades

\begin{tabular}{|l|c|c|c|c|c|c|c|c|c|r|}
\hline \multirow{2}{*}{ Atividades } & \multicolumn{7}{|c|}{ \% do custo dos recursos aplicado na atividade } & \multirow{2}{*}{ Custo da atividade } \\
\cline { 2 - 11 } & $\mathbf{1}$ & $\mathbf{2}$ & $\mathbf{3}$ & $\mathbf{4}$ & $\mathbf{5}$ & $\mathbf{6}$ & $\mathbf{7}$ & $\mathbf{8}$ & $\mathbf{9}$ & \\
\hline Pesquisa de obras & 40 & & & & & & & & & $\mathrm{R} \$ 31.416,80$ \\
\hline Resumo de obras & 10 & & & & 2 & & & & & $\mathrm{R} \$ 7.968,20$ \\
\hline Análise proj. e doc.. & & 4,5 & & & & & & & & $\mathrm{R} \$ 2.168,78$ \\
\hline Elaborar quantitativo & & & & & 42,5 & & & 400 & & $\mathrm{R} \$ 10.737,50$ \\
\hline Análise de quantit. & & 6 & & & & & & & & $\mathrm{R} \$ 2.891,70$ \\
\hline Orçamento & & 23,5 & & & 4,5 & 200 & & & & $\mathrm{R} \$ 17.982,33$ \\
\hline Lista de orçamento & & & & & 8,5 & & & & & $\mathrm{R} \$ 484,50$ \\
\hline Buscar fornecedores & & & 50 & & & & & & 50 & $\mathrm{R} \$ 15.520,00$ \\
\hline Cotar & & & 50 & & & & & & 50 & $\mathrm{R} \$ 15.520,00$ \\
\hline
\end{tabular}




\begin{tabular}{|l|r|r|l|l|l|l|l|l|l|r|}
\hline Elaborar relatório & & 7 & & & & & & & & $\mathrm{R} \$ 3.373,65$ \\
\hline Visitar o local & & 35 & & & 42,5 & & 150 & & & $\mathrm{R} \$ 50.449,74$ \\
\hline Acompanhar licit. & 25 & & & & & & & & & $\mathrm{R} \$ 19.635,50$ \\
\hline Reunião & 5 & 12 & & 34 & & & & & & $\mathrm{R} \$ 14.329,06$ \\
\hline Elaborar documentação & & 12 & & 48,5 & & & & & & $\mathrm{R} \$ 12.371,64$ \\
\hline Certidões & & & & 4 & & & & & & $\mathrm{R} \$ 543,36$ \\
\hline Atualizar certidões & & & & 13,5 & & & & & & $\mathrm{R} \$ 1.833,84$ \\
\hline Entrega envelope & 20 & & & & & & 150 & & & $\mathrm{R} \$ 46.867,39$ \\
\hline Totais & 100 & 100 & 100 & 100 & 100 & 200 & 300 & 400 & 100 & $\mathrm{R} \$ 254.093,97$ \\
\hline
\end{tabular}

Tabela 4 - Direcionamento dos custos das atividades às obras

\begin{tabular}{|c|c|c|c|c|c|c|}
\hline Atividade & Direcionador & Custo Atividade & $\begin{array}{l}\text { Custo } \\
\text { Unitário }\end{array}$ & \begin{tabular}{|c|} 
Quantitativo \\
de Obras com \\
licitação \\
vencida \\
\end{tabular} & $\begin{array}{c}\text { Quantitativo de } \\
\text { Obras - licitações } \\
\text { concorridas e } \\
\text { perdidas } \\
\end{array}$ & $\begin{array}{c}\text { Quantitativo de } \\
\text { Obras - } \\
\text { licitações } \\
\text { desistidas } \\
\end{array}$ \\
\hline Pesquisa de Obras & $\mathrm{n}^{\circ}$ ocorrência & $\mathrm{R} \$ \quad 31.416,80$ & $\mathrm{R} \$ 628,34$ & 19 und & 22 und & 9 und \\
\hline Resumo obras de interesse & Preço orçado (Mi) & $\mathrm{R} \$ \quad 7.968,20$ & $\mathrm{R} \$ 199,70$ & $17,23 \mathrm{mi}$ & $16,83 \mathrm{mi}$ & $5,84 \mathrm{mi}$ \\
\hline Verificação Proj./Doc. & $\mathrm{m}^{2}$ de obra & $\mathrm{R} \$ \quad 2.168,78$ & $\mathrm{R} \$ \quad 0,04$ & $19690 \mathrm{~m}^{2}$ & $23347 \mathrm{~m}^{2}$ & $18823 \mathrm{~m}^{2}$ \\
\hline Elaborar Quantitativo & $\mathrm{m}^{2}$ de obra & $\mathrm{R} \$ \quad 10.737,50$ & $\mathrm{R} \$ \quad 0,17$ & $19691 \mathrm{~m}^{2}$ & $23347 \mathrm{~m}^{2}$ & $18823 \mathrm{~m}^{2}$ \\
\hline Avaliar Quantitativo & $\mathrm{m}^{2}$ de obra & $\mathrm{R} \$ \quad 2.891,70$ & $\mathrm{R} \$ \quad 0,05$ & $19692 \mathrm{~m}^{2}$ & $23347 \mathrm{~m}^{2}$ & $18823 \mathrm{~m}^{2}$ \\
\hline Orçamento & $\mathrm{m}^{2}$ de obra & $\mathrm{R} \$ \quad 17.982,33$ & $\mathrm{R} \$ \quad 0,29$ & $19693 \mathrm{~m}^{2}$ & $23347 \mathrm{~m}^{2}$ & $18823 \mathrm{~m}^{2}$ \\
\hline Lista de Orçamento & $\mathrm{m}^{2}$ de obra & 484,50 & $\mathrm{R} \$ \quad 0,01$ & $19694 \mathrm{~m}^{2}$ & $23347 \mathrm{~m}^{2}$ & $18823 \mathrm{~m}^{2}$ \\
\hline Buscar Fornecedores & $\mathrm{m}^{2}$ de obra & $\mathrm{R} \$ \quad 15.520,00$ & $\mathrm{R} \$ \quad 0,25$ & $19695 \mathrm{~m}^{2}$ & $23347 \mathrm{~m}^{2}$ & $18823 \mathrm{~m}^{2}$ \\
\hline Cotar & $\mathrm{m}^{2}$ de obra & $\mathrm{R} \$ \quad 15.520,00$ & $\mathrm{R} \$ \quad 0,25$ & $19696 \mathrm{~m}^{2}$ & $23347 \mathrm{~m}^{2}$ & $18823 \mathrm{~m}^{2}$ \\
\hline Relatório & $\mathrm{n}^{\circ}$ ocorrência & $\mathrm{R} \$ \quad 3.373,65$ & $\mathrm{R} \$ 67,47$ & 19 und & 22 und & 9 und \\
\hline Visita obras & Dist. Da obra $(\mathrm{Km})$ & $\mathrm{R} \$ \quad 50.449,74$ & $\mathrm{R} \$ \quad 4,19$ & $4759 \mathrm{Km}$ & $5761 \mathrm{Km}$ & $1526 \mathrm{Km}$ \\
\hline Acompanhar Licitações & $\mathrm{n}^{\circ}$ ocorrência & $\mathrm{R} \$ 19.635,50$ & $\mathrm{R} \$ 392,71$ & 19 und & 22 und & 9 und \\
\hline Reunião & $\mathrm{n}^{\circ}$ ocorrência & $\mathrm{R} \$ \quad 14.329,06$ & $\mathrm{R} \$ 286,58$ & 19 und & 22 und & 9 und \\
\hline Elaborar Doc. & $\mathrm{n}^{\circ}$ ocorrência & $\mathrm{R} \$ \quad 12.371,64$ & $\mathrm{R} \$ 301,75$ & 19 und & 22 und & 0 und \\
\hline Certidões & $\mathrm{n}^{\circ}$ ocorrência & 543,36 & $\mathrm{R} \$ 13,25$ & 19 und & 22 und & 0 und \\
\hline Atualizar certidões & $\mathrm{n}^{\circ}$ ocorrência & $\mathrm{R} \$ \quad 1.833,84$ & $\mathrm{R} \$ 44,73$ & 19 und & 22 und & 0 und \\
\hline Entregar Envelopes & Dist. Da obra $(\mathrm{Km})$ & $\mathrm{R} \$ \quad 46.867,39$ & $\mathrm{R} \$ \quad 3,89$ & $4759 \mathrm{Km}$ & $5761 \mathrm{Km}$ & $1526 \mathrm{Km}$ \\
\hline \multicolumn{2}{|l|}{ TOTAL } & $\mathrm{R} \$ 254.093,97$ & - & - & - & - \\
\hline
\end{tabular}

Observações:

(a) Nas atividades de Busca de fornecedores e Cotação, como os dados de quantitativos de itens a serem orçados não existem, os direcionadores foram utilizados $\mathrm{em}^{2}$ de obra, assumindo que quanto maior a obra, maior o $\mathrm{n}^{\circ}$ de itens a serem orçados.

(b) As atividades de Elaboração de documentos, Emissão de Certidões e Atualização de Certidões têm apenas 41 unidades, pois só ocorrem se a empresa participar da licitação, neste caso, no período houve 9 desistências

Conhecido o custo unitário das atividades e o volume de atividades demandado por cada perfil de participação da empresa, torna-se possível obter o custo final do período por atividade desempenhada, conforme a tabela 5.

\section{Tabela 5 - Resumo da atribuição de custos baseada em atividades}

\begin{tabular}{|l|c|c|c|c|c|}
\hline \multicolumn{1}{|c|}{ Perfil de obra } & $\begin{array}{c}\mathbf{N}^{\circ} \\
\text { obras }\end{array}$ & $\begin{array}{c}\text { Dist. obra } \\
(\mathbf{K m})\end{array}$ & $\begin{array}{c}\text { Área da } \\
\text { obra (m) }\end{array}$ & $\begin{array}{c}\text { Preço Orçado } \\
(\mathbf{R} \mathbf{)})\end{array}$ & $\begin{array}{c}\text { Custo por } \\
\text { perfil }\end{array}$ \\
\hline Lic. Vencida & 19 & 4759 & 19690 & $\mathrm{R} \$ 17,23$ & $\mathrm{R} \$ 95.636,04$ \\
\hline Lic. Concorridas e vencida & 22 & 5761 & 23347 & $\mathrm{R} \$ 16,83$ & $\mathrm{R} \$ 112.716,59$ \\
\hline Lic. Avaliadas e desistidas & 9 & 1526 & 18823 & $\mathrm{R} \$ 5,84$ & $\mathrm{R} \$ 45.471,14$ \\
\hline TOTAL & 50 & 12046 & 61186 & $\mathrm{R} \$ 39,90$ & $\mathrm{R} \$ 254.093,77$ \\
\hline
\end{tabular}




\section{DISCUSSÃO}

Uma comparação entre os resultados obtidos pela aplicação do método $\mathrm{ABC}$ e aqueles resultantes do sistema existente de custeio indica diferenças bastante significativas entre os valores por eles obtidos (Tabela 6), podendo-se afirmar que parte dos custos das licitações com efetiva participação da empresa era absorvido (subsidiado) pelas obras onde houve desistência. Isto pode levar a crença que a desistência implica uma penalidade elevada, fazendo com que a empresa postergue essa decisão para momentos mais avançados do processo, contribuindo para aumentar custos totais.

Tabela 6 - Comparação entre sistema existente e custeio ABC

\begin{tabular}{|l|r|r|}
\hline \multicolumn{1}{|c|}{ Perfil de participação } & Custo - Método Tradicional & Custo - Método ABC \\
\hline Licitação Vencida & $\mathrm{R} \$ 80.877,95$ & $\mathrm{R} \$ 95.636,08$ \\
\hline Lic. Concorridas e perdidas & $\mathrm{R} \$ 95.899,32$ & $\mathrm{R} \$ 112.716,28$ \\
\hline Lic. Avaliadas e desistidas & $\mathrm{R} \$ 77.316,70$ & $\mathrm{R} \$ 45.741,61$ \\
\hline TOTAL & $\mathrm{R} \$ 254.093,97$ & $\mathrm{R} \$ 254.093,97$ \\
\hline
\end{tabular}

Porém, mais importante que a determinação dos custos de cada um dos tipos de obras é o suporte que o custeio $\mathrm{ABC}$ proporciona para questionar as razões para ocorrência de tais custos. Uma primeira avaliação dos custos médios por obra para cada um dos tipos estudados (dividindo-se os custos ABC da Tabela 6 pelo número de obras, indicado na Tabela 5) mostra que os mesmos são muito semelhantes: $\mathrm{R} \$ 5.033,48$ para licitações vencidas, R\$ 5.123,47 para licitações perdidas e $\mathrm{R} \$ 5.082,40$ para licitações abandonadas. Isto poderia indicar que uma forma mais simples de se dividir os custos pelas obras poderia ser atribuindo um mesmo valor médio por obra.

No entanto, uma análise mais aprofundada desses tipos de obras em termos dos direcionadores de custos utilizados mostra que as licitações abandonadas são substancialmente distintas das demais: sua distância média é menor (170km, comparados com $250 \mathrm{~km}$ das licitações vencidas e $262 \mathrm{~km}$ das perdidas), ao passo que seu tamanho médio é substancialmente maior $(2091 \mathrm{~m} 2$, comparados com $1036 \mathrm{~m} 2$ das licitações vencidas e $1061 \mathrm{~m} 2$ das licitações perdidas). Este fato traz como consequência que os custos associados com a distância são menores que os demais tipos, ao passo que os custos associados ao tamanho são praticamente o dobro, o que justifica a semelhança do custo médio já que uma variação atenua ou neutraliza a outra.

Mais importante que isto é a possibilidade que o custeio $\mathrm{ABC}$ proporciona de questionar o porquê das licitações com desistência serem tão distintas das demais. Uma melhor compreensão destas causas poderia permitir que a desistência ocorresse já nos momentos iniciais do processo, evitando-se assim a ocorrência de custos desnecessários.

Neste sentido, cabe ainda uma observação final: o modelo $\mathrm{ABC}$ adotado neste estudo parte da premissa que todas as obras utilizam todas as atividades, o que pode não ser correto no caso das obras com desistência, caso esta desistência ocorra nos momentos iniciais, como mencionado acima. Isto indica que a desistência nos momentos iniciais poderia contribuir ainda mais para a redução dos custos, bem além do indicado pelo custeio ABC.

\section{CONSIDERAÇÕES FINAIS}

Este trabalho buscou demonstrar a aplicação do custeio $\mathrm{ABC}$ para o processo de 
alocação de custos do processo de elaboração de propostas de preços para licitações. $\mathrm{O}$ estudo revela que a aplicação do método $\mathrm{ABC}$ pode trazer resultados altamente relevantes não apenas para a alocação de custos indiretos aos objetos de custo, mas também no sentido de proporcionar um melhor conhecimento do processo envolvido, e permitir um questionamento das causas desses custos. No caso estudado, o custeio ABC mostrou que as licitações onde ocorreu desistência por parte da empresa envolviam obras muito distintas das demais em termos do tamanho da obra e sua distância, apesar da semelhança existente em termos de seus custos médios. Isto sugere um questionamento e estudo mais aprofundado das razões pelas quais ocorrem as desistências, e até que ponto tais razões podem ser associadas com as diferenças identificadas.

\section{REFERÊNCIAS}

ALTOUNIAN, Cláudio Sarian. Obras públicas: licitação, contratação, fiscalização e utilização. 3. ed. rev., atual. e ampl. Belo Horizonte: FORUM, 2012. 413 p.

BRASIL. Lei No 8.666, de 21 de junho de 1993. Regulamenta o art. 37, inciso XXI, da Constituição Federal, institui normas para licitações e contratos da Administração Pública e dá outras providências. Disponível em http://www.planalto.gov.br/ccivil_03/leis/18666cons.htm

BRIMSON, James A. Contabilidade por Atividades. São Paulo: Atlas, 1994.

CALESSO, DANIEL B. Análise de custos com foco em método ABC, Variável e Absorção. 2010. TCC (Bacharelado em Ciências Contábeis) - Universidade Federal do Rio Grande do Sul, Porto Alegre, 2010.

JOHNSON, H. T.; KAPLAN, R. S. Contabilidade gerencial: A restauração da relevância da contabilidade nas empresas. Rio de Janeiro: Campus, 1993.

HORNGREN, CHARLES T.; DATAR. Srikant M.; RAJAN, Madhav V. Cost accounting: a managerial emphasis. 14th ed. Boston, MA: Prentice Hall, 2012.

KAPLAN, R.S.; COOPER, R. Cost and Effect: Using Integrated Cost Systems to Drive Profitability and Performance. Boston, MA: Harvard Business School Press, 1998

KHOURY, Carlos Y.; Ancelevicz, Jacob. Controvérsias Acerca do Sistema de Custos ABC. RAE - Revista de Administração de Empresas, 2000

KIM, YONG-WOO; BALLARD, GLEEN. Activity-Based Costing and its Application to Lean Construction. 9th annual conference International Group Of Lean Construction (IGLC). Singapura: National University of Singapore, August, 2001

MARTINS, E. Contabilidade de custos. São Paulo: Atlas, 2003.

MAUAD, LUIZ G. A. E PAMPLONA, EDSON O. O Custeio ABC em empresas de serviços: características observadas na implantação em uma empresa do setor. IX Congresso Brasileiro de Custos. São Paulo, 2002.

NAKAGAWA, MASAYUKI. ABC - Custeio Baseado em Atividades. 2. ed. São Paulo: Atlas, 2001. 95 p.

ROCHA, JOÃO G. C.; DIAS, JOSÉ C. S.; COPPINI, NIVALDO L. ABC - Custeio Baseado em Atividades: uma Aplicação em Empresas de Construção Civil, Revista Ciência \& Tecnologia, 14, 1999. p. 31-44. 